\title{
Heterópteros acuáticos y semiacuáticos asociados al bosque seco del Patía, suroeste de Colombia
}

\author{
Aquatic and semiaquatic Heteropterans associated with dry \\ forest in Patía, southwestern Colombia
}

\author{
Dora Nancy Padilla Gil
}

\section{Resumen}

Los ecosistemas secos del Patía, en los Departamentos de Cauca y Nariño, son unos de los más degradados y altamente vulnerables frente al cambio climático en Colombia, y son áreas prioritarias para conservación. Determinamos las especies de heterópteros acuáticos y semiacuáticos, Nepomorpha y Gerromorpha, que habitan en los ecosistemas acuáticos de este bosque seco, basado en la revisión de publicaciones y de la colección de Entomología PSO-CZ. Se registraron 27 especies: Gerromorpha, 21 especies y Nepomorpha, 6 especies.

Palabras clave. Nadadores de espalda. Neotrópico. Patinadores. Sudamérica.

\begin{abstract}
The dry forests of the Patía River, in the Departments of Cauca and Nariño, are some of the most degraded and highly vulnerable ecosystems facing climate change in Colombia, and they are considered high priority areas for conservation. We identified aquatic and semiaquatic heteropterans (Nepomorpha and Gerromorpha) that inhabit the aquatic ecosystems in these dry forests, based on literature and on the PSO-CZ Entomological Collection. There were 27 species: Gerromorpha, 21 species and Nepomorpha, 6 species.
\end{abstract}

Key word. Back swimmers. Neotropical Region. South America. water striders.

\section{Introducción}

El río Patía es el segundo de mayor caudal del Pacífico, después del río San Juan. De los ecosistemas Secos del Patía queda menos del $0.6 \%$ de la cobertura original, y el tamaño del área de este bosque seco es de 28 ha (García et al., 2014; MADS \& PNNC, 2019).

El valle del Patía tiene una precipitación promedio anual entre 800 y 1000 mm anuales; la vegetación corresponde a bosque muy seco tropical y bosque seco premontano, el primero en áreas más bajas y el segundo en las faldas de las cordilleras (Ramírez-Padilla et al., 2018).
La flora de los ecosistemas de bosque seco del Patía ha sido estudiada principalmente en el departamento del Cauca y escasamente en Nariño (Vergara, 2015; Bolaños, 2016; Ramírez et al., 2015, 2018; Eraso et al, 2017). En fauna, se han estudiado principalmente aves y mamíferos (Ramírez et al., 2010; Ayerbe-Quiñones \& López-Ordoñez, 2011; Calderón, 2012; Villegas, 2016); sin embargo, los insectos acuáticos y semiacuáticos no han sido objeto de estudio.

El bosque seco del río Patía está representado por el orobioma azonal y sus ecosistemas de aguas continentales, arbustales, vegetación secundaria y zonas desnudas. Estos bosques han recibido fuerte intervención antrópica, deforestación, erosión de suelos, desertificación, 
minería, e infraestructura humana, lo cual los hace vulnerables ante el cambio climático y por esto se consideran como un área prioritaria para efectos de conservación y manejo sostenible (Pizano et al., 2017; MADS \& PNNC, 2019).

El valle seco del Patía está irrigado por el río Patía y sus principales afluentes son los ríos Guachicono, San Jorge, San Pablo, Juanambú, Iscuandecito, y Piusbí. Los ecosistemas acuáticos lénticos y lóticos de esta cuenca son el hábitat de heterópteros acuáticos y semiacuáti$\cos$, que hacen parte integral de las cadenas tróficas y son reconocidos por su potencial como indicadores de cambio climático y de metales pesados y como agentes de control biológico de mosquitos de importancia médica (Nummelin et al., 1998; Yanoviak, 1999; Chen et al., 2012; Padilla-Gil, 2012).

Los heterópteros acuáticos pertenecen al infraorden Nepomorpha y los semiacuáticos a Gerromorpha. En los ecosistemas andinos de Colombia se ha estudiado la diversidad y abundancia de los gerromorfos en departamento del Tolima y en el piedemonte Amazónico y del Pacífico (Parra-Trujillo et al., 2014; Padilla-Gil, 2016a; 2019a, b).

Dada la importancia del bosque seco tropical del suroeste de Colombia y de los heterópteros acuáticos y semiacuáticos como biota integral de los cuerpos de agua de estos ecosistemas, se plantea este trabajo que tiene como objetivo dar a conocer esta biota con el fin de ampliar el conocimiento taxonómico y biogeográfico de los gerromorfos y nepomorfos de los Andes de los ecosistemas secos del Patía de los departamentos de Cauca y Nariño.

\section{Materiales y Métodos}

Se revisaron las publicaciones con registros de recolección en esta área de bosque seco de los departamentos de Cauca y Nariño (Padilla-Gil \& Nieser, 2003; PadillaGil, 2011; 2013a, b; 2016b, 2019b) y los heterópteros acuáticos y semiacuáticos depositados en la colección de entomología de la Universidad de Nariño (PSO-CZ).

\section{Ubicación de los Ecosistemas Secos del Patía}

Los ecosistemas secos del Patía cubren el área geográfica de seis municipios en el departamento del Cauca y 15 en el departamento de Nariño (MADS \& PNNC, 2019). Otros fragmentos de bosque seco del Patía adicionados para la distribución geográfica de los heterópteros acuáticos y semiacuáticos son los parches que se encuentran en los municipios de Génova (Colón), con vegetación de bosque seco montano bajo, y San Pablo, con bosque seco premontano.

\section{Resultados}

Los ecosistemas secos del Patía presentan 27 especies: Gerromorpha, cuenta con 3 familias, 6 géneros y 21 especies; los Nepomorpha: 4 familias, 6 géneros y 6 especies (Tabla 1). La familia Veliidae está representada con el $59 \%$ del total de especies; por otra parte Gerridae supera a Veliidae en el número de géneros. Rhagovelia es el género que cuenta con mayor número de especies, con $48 \%$ del total, seguido por Microvelia y Trepobates.

Tabla 1. Lista de heterópteros acuáticos y semiacuáticos de ecosistemas secos del Patía, Colombia; distribución por municipios y altitudes.

\begin{tabular}{lccc}
\hline \multicolumn{1}{c}{ Taxon } & $\begin{array}{c}\text { Altitud } \\
(\mathbf{m} \text { s.n.m. })\end{array}$ & Municipio & Departamento \\
\hline Gerromorpha & & & \\
\hline Hydrometridae & 600 & Patía & Cauca \\
\hline Hydrometra caraiba Guérin-Méneville, 1856 & $1750-1788$ & Timbío & Cauca \\
\hline Gerridae & $2420-2455$ & Buesaco & Nariño \\
\hline Eurygerris fuscinervis (Berg, 1898) & 1800 & Chachagüí & Nariño \\
\hline & 2180 & La Florida & Nariño \\
\hline
\end{tabular}




\begin{tabular}{|c|c|c|c|}
\hline Taxon & $\begin{array}{c}\text { Altitud } \\
\text { (m s.n.m.) }\end{array}$ & Municipio & Departamento \\
\hline & 2162 & La Cruz & Nariño \\
\hline & 1727 & La Unión & Nariño \\
\hline & 1500 & Policarpa & Nariño \\
\hline & 1500 & Taminango & Nariño \\
\hline Limnogonus franciscanus (Stal, 1859) & 600 & Patía & Cauca \\
\hline Trepobates taylori (Kirkaldy, 1999) & $572-600$ & Patía & Cauca \\
\hline Trepobates trepidus Drake \& Harris, 1928 & 600 & Patía & Cauca \\
\hline \multicolumn{4}{|l|}{ Veliidae } \\
\hline Microvelia acantha (Padilla-Gil, 2013) & 769 & Mercaderes & Cauca \\
\hline Microvelia fanera (Padilla-Gil, 2013) & 1729 & Buesaco & Nariño \\
\hline Microvelia longipes Uhler, 1894 & 572 & Mercaderes & Cauca \\
\hline Rhagovelia buesaquensis Padilla-Gil, 2015* & 1900 & Buesaco & Nariño \\
\hline \multirow[t]{3}{*}{ Rhagovelia cali Polhemus, 1997} & 1970 & Buesaco & Nariño \\
\hline & 1929 & Génova & Nariño \\
\hline & 2200 & La Florida & Nariño \\
\hline \multirow[t]{2}{*}{ Rhagovelia carina Padilla-Gil, 2015} & 769 & Mercaderes & Cauca \\
\hline & 1788 & Timbío & Cauca \\
\hline \multirow[t]{2}{*}{ Rhagovelia cauca Polhemus, 1997} & 769 & Mercaderes & Cauca \\
\hline & $1750-1788$ & Timbío & Cauca \\
\hline Rhagovelia chachigi Padilla-Gil, 2019* & 1655 & Chachagüí & Nariño \\
\hline Rhagovelia cimarrona Padilla-Gil, 2011* & 1650 & Chachagüí & Nariño \\
\hline \multirow[t]{2}{*}{ Rhagovelia gastrotricha Padilla-Gil, 2011} & 572 & Mercaderes & Cauca \\
\hline & 586 & Patía & Cauca \\
\hline Rhagovelia guachiconoense Padilla-Gil, 2019* & 769 & Mercaderes & Cauca \\
\hline Rhagovelia longipes Gould, 1931 & $723-725$ & Mercaderes & Cauca \\
\hline Rhagovelia mirabilis Padilla-Gil, 2019* & 1300 & Buesaco & Nariño \\
\hline Rhagovelia pajajoyi Padilla-Gil, 2019* & 1950 & Buesaco & Nariño \\
\hline Rhagovelia policarpa Padilla-Gil, 2019* & 1000 & Policarpa & Nariño \\
\hline Rhagovelia villamoreno Padilla-Gil, 2019* & 2431 & Buesaco & Nariño \\
\hline \multicolumn{4}{|l|}{ Nepomorpha } \\
\hline \multicolumn{4}{|l|}{ Corixidae } \\
\hline Centrocorisa kollari (Fieber, 1851) & 572 & Patía & Cauca \\
\hline \multicolumn{4}{|l|}{ Notonectidae } \\
\hline Buenoa crassipes (Champion, 1901) & 572 & Patía & Cauca \\
\hline
\end{tabular}




\begin{tabular}{lccc}
\hline \multicolumn{1}{c}{ Taxon } & $\begin{array}{c}\text { Altitud } \\
(\mathbf{m} \text { s.n.m. })\end{array}$ & Municipio & Departamento \\
\hline Notonecta indica Linnaeus, 1771 & 599 & Patía & Cauca \\
\hline Gelastocoridae & & & \\
\hline Gelastocoris oculatus (Fabricius, 1798) & 586 & Mercaderes & Cauca \\
\hline & 587 & Patía & Cauca \\
\hline Nerthra ater (Melin, 1929) & 1683 & San Pablo & Nariño \\
\hline Naucoridae & & & \\
\hline Pelocoris poeyi (Guérin-Méneville, 1835) & 600 & Patía & Cauca \\
\hline
\end{tabular}

* Registros únicos para esta área geográfica

La mayoría de ecosistemas acuáticos muestreados fueron lóticos, ríos y quebradas; esto se ve reflejado en la diversidad y distribución de los gerromorfos y algunos nepomorfos de las orillas, como los gelastocóridos. La especie con mayor número de registros y de distribución geográfica fue Eurygerris fuscinervis y Rhagovelia cali, seguidas por tres especies de Rhagovelia y G. oculatus, con dos registros; el resto de especies cuentan con un solo registro.

Ocho especies presentan distribución restringida a estos ecosistemas secos (señaladas con asterisco en la tabla 1). Rhagovelia cauca y Microvelia fanera, también se encuentran en otros bosques secos como el Valle Alto del río Magdalena (Parra-Trujillo et al., 2014; Padilla-Gil, 2016a).

\section{Discusión}

Los heterópteros acuáticos y semiacuáticos de los bosques secos son poco conocidos. Moreno et al. (2018) estudiaron las chinches acuáticas del municipio de Toluviejo (Sucre), en la región Caribe de Colombia; la composición a nivel de especies, tanto de Gerromorpha como de Nepomorpha, es diferente a la registrada para los ecosistemas secos del Patía; el tipo de vegetación también es diferente: mientras los ecosistemas secos del Patía corresponden a bosque muy seco tropical, bosque seco premontano (Ramírez-Padilla et al., 2018) y bosque seco montano bajo; el área de Toluviejo corresponde a bosque seco tropical. Es posible que la topografía, heterogeneidad de hábitats, y ecología puedan estar relacionadas con estas diferencias.

Ocho especies de Rhagovelia permanecen con rango de distribución restringido a estos ecosistemas acuáticos del Patía (Tabla 1); otras especies, como Rhagovelia cauca y Rhagovelia cali, se extienden hasta Ecuador (Padilla-Gil \& Moreira, 2013); se necesitan trabajos futuros, principalmente en los bosques secos de Ecuador, que permitan precisar los rangos geográficos de estas especies de Rhagovelia. Por otra parte, E. fuscinervis no sólo es muy abundante en los cuerpos de agua del Patía, sino que su rango geográfico es muy amplio, tanto en Colombia como en América, desde México hasta Argentina (Padilla-Gil \& Arcos, 2010).

La variación altitudinal cambia drásticamente, tanto al norte del valle del Patía, en el departamento del Cauca, como en el sur de este valle, en Nariño. En este último hay varios municipios sin registros, por lo que la lista de especies presentada aquí es preliminar, y es necesario ampliar el área, como el tiempo de muestreo, en los ecosistemas secos del Patía.

\section{Referencias}

Ayerbe-Quiñones, F. \& López-Ordoñez, J. P. (2011). Adiciones a la avifauna del valle del río Patía, un área interandina en el suroccidente de Colombia. Boletín SAO, 20(2), 1-17.

Bolaños, A. (2016). Caracterización de la vegetación en una zona delimitada en Cumbitara, La Llanada, Policarpa y los Andes Sotomayor (Nariño), como insumo para la identificación de oportunidades de conservación para la recuperación de los servicios ecosistémicos de bosque seco. v1.4. Corporación Paisajes Rurales. Dataset/Ocurrence. https:// bit.ly/3baL905

Calderón, L. J. J. (ed) (2012). Charmolán: Rostros y Retos de la Conservación. San Juan de Pasto, Nariño, Colombia: Universidad de Nariño. 227 pp. 
Chen, L., Damgaard, J. \& Garrouste, R. (2012). The sea-skater Halobates (Heteroptera: Gerridae) probable cause for extinction in the Mediterranean and potential for re-colonisation following climate change. Aquatic Insects, 34(sup1), 45-55.

Eraso, E. A. L., Gamboa, B. J. H. \& Ramírez, P. B. R. (2017). Estructura y composición vegetal de un fragmento de bosque seco tropical, en el municipio del Patía, Cauca. Revista Novedades Colombianas, 12(1), 25-37.

García, H., Corzo, G., Isaacs, P. \& Etter, A. (2014). Distribución y estado actual de los remanentes del bioma de bosque seco tropical en Colombia; insumos para su gestión, capítulo 8. En: Pizano, C. \& García, H. (Eds.). El bosque seco tropical en Colombia. Pp: 228251. Bogotá, Colombia: Instituto de Investigación en Recursos Biológicos Alexander von Humboldt.

Ministerio de Ambiente y Desarrollo Sostenible (MADS) \& Parques Nacionales Naturales de Colombia (PNNC). (2019). Portafolio de nuevas áreas y ampliaciones del ámbito de gestión nacional, liderado por Parques Nacionales Naturales de Colombia. http://www.parquesnacionales.gov.co/portal/es/

Moreno, C., Molina, W., Barbosa, J. F. \& Moreira, F. F. F. (2018). Aquatic and semiaquatic bugs (Insecta, Hemiptera, Heteroptera) from Toluviejo Municipality, Sucre Department, Caribbean region of Colombia. Check List, 14(6), 985-1002. https://doi.org/10.15560/14.6.985

Nummelin, M., Lodenius, M. \& Tulisalo, E. (1998). Water striders (Heteroptera: Gerridae) as bioindicators of heavy metal pollution. Entomologica Fennica, 8,185-191.

Padilla-Gil, D. N. (2011). Ten new species of Rhagovelia in the $R$. angustipes complex from Colombia (Heteroptera: Veliidae). Aquatic insects, 33(3), 203-231. https://doi.org/10.1080/01650424.2011.597404

Padilla-Gil, D. N. (2012). Los hemípteros acuáticos del municipio de Tumaco (Nariño, Colombia) Guía ilustrada. San Juan de Pasto, Nariño, Colombia: Universidad de Nariño. 88 pp.

Padilla-Gil, D. N. (2013a). Nuevos registros y ampliación de la distribución de Heterópteros acuáticos en Colombia (Hemiptera, Heteroptera). Acta Biológica Colombiana, 18(2), 391-400.

Padilla-Gil, D. N. (2013b). Two new species of Paravelia Breddin, 1898 (Hemiptera: Heteroptera: Veliidae) from Colombia, with a key to Colombian Paravelia species. Zootaxa, 3693(4), 491-502. https:// doi.org/10.11646/zootaxa.3693.43.5

Padilla-Gil, D. N. (2016a). Las chinches semi-acuáticas de la Reserva Natural Río Nambí (Nariño) Colombia. Acta Biológica Colombiana, 21(1), 201-206. https:// doi.org/10.15446/abc.v21n1.50001
Padilla-Gil, D. N. (2016b). Nuevos registros de Heteroptera (Hemiptera) acuáticos y semiacuáticos de Colombia. Biota Colombiana, 17(2), 39-46. https:// doi.org/10.21068/c2016.v17n02a04

Padilla-Gil, D. N. (2019a). Diversidad del género Rhagovelia (Hemiptera: Heteroptera: Veliidae) del piedemonte Andino-Amazónico (Putumayo, Colombia). Acta Biológica Colombiana, 24(1), 174-179. https://doi.org/10.15446/abc.v24n1.66352

Padilla-Gil, D. N. (2019b). Nuevas especies de Rhagovelia, Microvelia, Buenoa-Registros nuevos de otros heterópteros de Colombia (Gerromorpha, Nepomorpha, Leptopodomorpha). Beau Bassin: Editorial Académica Española. 242 pp.

Padilla-Gil, D. N. \& Arcos, O. (2010). Estadios y variación temporal de Eurygerris fuscinervis (Heteroptera: Gerridae) en los Andes de Colombia. Revista Colombiana de Entomología, 36(2), 300-303.

Padilla-Gil, D. N. \& Moreira, F.F.F. (2013). Checklist, taxonomy and distribution of the Rhagovelia Mayr, 1865 (Hemiptera: Veliidae) of the Americas. Zootaxa, 3640(3), 409-424. http://doi.org/10.11646/zootaxa.3640.3.5

Padilla-Gil, D. N. \& Nieser, N. (2003). Nueva especie de Tachygerris y nuevos registros de colecta de las Gerridae (Hemiptera: Heteroptera) de Colombia. Actualidades Biológicas, 25(78), 39-49.

Parra-Trujillo, Y. T., Padilla-Gil, D. N. \& Reinoso, G. (2014). Diversidad y distribución de Rhagovelia (Hemiptera, Veliidae) del departamento del Tolima. Revista de la Asociación Colombiana de Ciencias Biológicas, 26, 82-88.

Pizano, C., González-M, R., Hernández-Jaramillo, A. \& García, H. (2017). Agenda de investigación y monitoreo en bosques secos de Colombia (20132015): fortaleciendo redes de colaboración para su gestión integral en el territorio. Biodiversidad en la Práctica, 2(1), 48-86.

Ramírez-Chaves, H., Ayerbe-Quiñones, F. \& Mejía-Egas, O. (2010). Mamíferos de la cuenca alta del Río Patía en el departamento del Cauca, Colombia. Boletín Científico Museo de Historia Natural Universidad de Caldas, 14(1), 92-113.

Ramírez-Padilla, B. R., Macías-Pinto, D. J. \& VaronaBalcázar, G. (2015). Lista comentada de plantas vasculares del valle seco del río Patía, suroccidente de Colombia. Biota Colombiana, 16(2), 1-50.

Ramírez-Padilla, B. R., Solarte-Téllez, V. \& RamírezBurbano, P. A. (2018). Musgos del valle seco del Patía, suroccidente de Colombia; riqueza, ecología y biogeografía. Biota Colombiana, 19(2), 1-11. http://doi.org/10.21068/c2018.v19n02a01 
Vergara, V. H. (2015). Patrones de la vegetación y tipos de usos de la tierra en el valle del Patía. Colombia Forestal, 18(1), 25-45. http://doi.org/10.14483/udistrital.jour.colomb.

Villegas, M. A. (2016). Caracterización de la avifauna en una zona delimitada entre los municipios de Cumbitara, La Llanada, Policarpa y Los Andes
Sotomayor (Nariño). v.2.3. Corporación Paisajes Rurales. Dataset/Occurrence. http://doi.org/10.15472/4xlwgt

Yanoviak, S. P. (1999). Distribution and abundance of Microvelia cavicola Polhemus (Heteroptera: Veliidae) on Barro Colorado Island, Panama. Journal of the New York Entomological Society, 107, 38-45.
Dora Nancy Padilla Gil

Universidad de Nariño

San Juan de Pasto, Nariño

dnpadilla@udenar.edu.co

https://orcid.org/0000-0002-9960-6103
Heterópteros acuáticos y semiacuáticos asociados al bosque seco del Patía, suroeste de Colombia

Citación del artículo: Padilla-Gil, D. N. (2020). Heterópteros acuáticos y semiacuáticos asociados al bosque seco del Patía, suroeste de Colombia. Biota colombiana, 21(2), 15-20. DOI: 10.21068/ c2020.v21n02a01.

Recibido: 15 de enero 2020

Aceptado: 14 de mayo 2020 